Marija Mojca Peternel* UDK 070(497.4=112.2) "1948"

Universität Ljubljana

DOI: 10.4312/linguistica.59.1.221-233

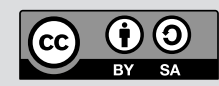

\title{
DEUTSCH IN DER PRESSE UM 1848 IN DEN VON SLOWENEN BESIEDELTEN GEBIETEN: EINE EXEMPLARISCHE ANALYSE AM BEISPIEL VON LAIBACHER KIRCHENZEITUNG, CILLIER ZEITUNG UND SLOVENIENS BLATT
}

\section{EINLEITUNG}

Im Beitrag wird versucht, die durch die Revolution 1848/49 initiierten deutschen Zeitungen (das heißt neue Zeitungen) im Raum mit slowenischer Bevölkerung aus sprachlicher Perspektive näher zu erläutern und zu vergleichen. Da das Jahr 1848/49 durch die Revolution geprägt wurde und die Zeitungen Spiegel ihrer Zeit sind, trugen diese einen ausgeprägt politischen Charakter. Es sollte der Frage nachgegangen werden, in welchem Ausmaß die Sprache der neuen Zeitungen dadurch geprägt wurde. Auch wird versucht, vor allem auf der morphologischen und syntaktischen Ebene Merkmale bzw. eventuelle Unterschiede im Sprachgebrauch auszumachen, und nach deren Ursache zu fragen. Die Analyse der neuen Zeitungen sollte schließlich bezeugen, wie die Zeitungssprache durch das Milieu beeinflusst wurde.

\section{PRESSE IM JAHR 1848}

Als am 15. März 1848 die Pressefreiheit mittels kaiserlichen Patents gewährt wurde, welches vor allem die Zusage enthielt: „Die Pressefreiheit ist durch unsere Erklärung der Aufhebung der Censur in derselben Weise gewahrt, wie in allen Staaten, wo sie besteht.“ (Zenker 1893: 12), wurde die Periode des sogenannten Zeitungssterbens (Pock 1925: 183) beendet und überall in der Monarchie erschienen täglich neue Zeitungen. In Wien vergrößerte sich so die Zahl der herausgegebenen Zeitungen von 42 auf 213 (Zenker 1893: 143-158).

Wie überall in der Monarchie, fand die Pressefreiheit auch in den Ländern mit slowenischer Bevölkerung ihren konkreten Niederschlag. Anfangs wurden nur verschiedene Flugblätter, später Zeitungen herausgegeben (Granda 1994: 231). Zuerst erschien in Celje (Cilli) das Cillier Wochenblatt (1. April 1848), das nach kurzer Zeit (4. Juli 1848) in Cillier Zeitung umbenannt wurde (vgl. Peternel 2006). Später folgte in Novo mesto (Neustadtl/Rudolfswert) die Zeitung Sloveniens Blatt (10. Mai 1848). Der Bedarf an politischen Mitteilungen und Berichten war sehr groß, und schließlich erschien auch in Ljubljana (Laibach) am 20. Juli 1848 die erste Nummer des Politischen Blattes als Extra-Beilage zur Laibacher Zeitung. Auch die hiesige Kirche begann im Sommer die slowenische Slovenski cerkveni časopis und die deutschsprachige Laibacher Kirchenzeitung, die am 6. Juli 1848 zum ersten Mal erschien, herauszugeben (vgl. Peternel 2015).

mojca.peternel@guest.arnes.si 
Neben den erwähnten Zeitungen erschienen in den Ländern mit slowenischer Bevölkerung auch neue slowenische Zeitungen: in Ljubljana (Laibach) die erste politische Zeitung in slowenischer Sprache Slovenija, in Celje (Cilli) die Celjske Slovenske Novine (Slowenische Cillier Zeitung) und noch einige weitere, wie die erste slowenische Zeitung in Triest Vedež.

In die sprachliche Analyse werden nur die oben genannten neuen (deutschen) Zeitungen aus Ljubljana (Laibach), Celje (Cilli) und Novo mesto (Neustadtl) einbezogen. Das Politische Blatt als Extra-Beilage zur Laibacher Zeitung wurde wegen ihres beschränkten Umfangs (nur zwei Seiten) aus der Analyse ausgeschlossen.

Vor der konkreten Sprachanalyse der durch die Revolution herausgegebenen Zeitungen sollte aber noch ein wichtiger Punkt angesprochen werden, der einen großen Einfluss auf das damalige Leben (und somit auch auf die Zeitungen) hatte. Es handelt sich um das berühmte slowenische Nationalprogramm (vgl. Granda 1999), dessen zweiter Punkt unter anderem die Vereinigung aller Slowenen in Österreich sowie die Einführung der slowenischen Sprache an Schulen und Behörden vorsah. Die Tatsache, dass die nach erlassener Pressefreiheit herausgegebenen Zeitungen im slowenischen Raum in deutscher Sprache abgefasst waren, scheint zunächst vielleicht unlogisch. Allerdings war es vielen klar, dass eine große Diskrepanz zwischen prinzipiellen Forderungen (im Nationalprogramm) und den realen, tatsächlichen Möglichkeiten bestand. Der so formulierte zweite Punkt des slowenischen Nationalprogramms ging unter anderem davon aus, dass Deutsch die Kommunikationssprache der damaligen Intellektuellen sowohl bei den Slawen der Habsburger Monarchie als vielfach auch außerhalb war und die Slowenen bildeten damals keine Ausnahme.

So benutzte z. B. sogar der größte slowenische Dichter France Prešeren, ${ }^{2}$ der auch in Wien studierte, in den Gesprächen (nicht nur) mit Anton Auersperg - Anastasius Grün ${ }^{3}$ lieber Deutsch.

Auch slowenische Beamte drängten nicht auf eine sofortige Einführung der slowenischen Amtssprache. Sie waren oft nicht fähig, die slowenische Sprache im öffentlichen oder sogar in ihrem privaten Leben uneingeschränkt zu verwenden.

\section{ANALYSE}

Vor der sprachlichen Analyse wird bei jeder Zeitung zunächst eine kurze Übersicht der Mitarbeiter bzw. Autoren der Leitartikel geboten, da eventuelle sprachliche Differenzen in den analysierten Zeitungen in engem Zusammenhang mit dem Bildungsgrad der Leitartikelautoren stehen.

\subsection{Die Laibacher Kirchenzeitung}

Der Redakteur beider kirchlichen Zeitungen war Janez Zlatoust Pogačar, ${ }^{4}$ der zuerst in Ljubljana und dann am Augustineum in Wien studierte (vgl. Ambrožič 2003). In dieser

2 France Prešeren (3. 12. 1800, Vrba/Velden - 8. 2. 1849, Kranj/Krainburg).

3 Anton Auersperg (11. 4. 1806, Ljubljana/Laibach - 12. 9. 1876, Graz). Vgl. Zalaznik/Granda 2009.

4 Janez Zlatoust Pogačar (22. 1. 1811 Vrba/Velden - 25. 1. 1884 Ljubljana/Laibach). 
Zeit wurde er mit bedeutenden Persönlichkeiten bekannt, unter anderem mit dem späteren Mitarbeiter der Laibacher Kirchenzeitung Dr. Alois Schlör ${ }^{5}$.

Die gesamte Laibacher Kirchenzeitung besteht fast ausschließlich aus Leitartikeln, deren Autoren auch slowenischer Herkunft waren: Bischof Anton Slomšek ${ }^{6}$, der spätere Bischof Jakob Maksimilian Stepišnik ${ }^{7}$ und der Priester und Historiker Peter Hitzinger ${ }^{8}$, der seine Artikel auch in anderen Presseerzeugnissen in Ljubljana (Laibach) veröffentlichte (Hitzinger 1848a: 484-485).

Neben dem schon erwähnten Wiener Professor Dr. Alois Schlör, dem vorbildlichen Redakteur der Laibacher Kirchenzeitung (Ambrožič 2003: 67), schrieben für die Laibacher Kirchenzeitung der spätere Gurker Bischof Wiery ${ }^{9}$ und der Historiker Leo Wonzhina ${ }^{10}$.

Dieser Überblick zeigt sehr deutlich, dass die Autoren der kirchlichen Zeitung fast ausschließlich hochgebildete Persönlichkeiten waren und davon ausgehend sollten die Leitartikel sprachlich auch fehlerfrei sein. Den Erwartungen entsprechend lassen sich inhaltlich anspruchsvollere Texte ausmachen, deren Syntax komplex auffällt. Hierzu ein Textauszug von Peter Hitzinger, der über die künftige Stellung der Kirche berichtet (Hitzinger 1848: 33):

Staat und Kirche haben neben einander in freundlicher Stellung zu stehen. Eines hat im Andern nicht aufzugehen, Eines über das Andere nicht zu herrschen; die Kirche wird dem Staat die geistliche Unterstützung nicht entsorgen dürfen, sobald der Staat in der Sphäre des Rechtes bleibt; dagegen wird die Kirche auch (wie der einzelne Staatsbürger) von Staat äußern Schutz fordern können.

Manchmal werden die Gedanken durch Semikolon getrennt, wie beispielsweise bei dem Redakteur der Laibacher Kirchenzeitung Janez Pogačar (Pogazhar1848a: 1):

Auch die Kirche kann von solchen stürmischen Bewegungen der Zeit nicht unberührt bleiben; sie lebt ja in und mit den Völkern und wird deshalb immer und überall in die Geschichte derselben hineingezogen; unter dem Schatten der Flügel des heiligen Geistes geht sie in alle Phasen der Weltgeschichte ein und bildet ihren innersten Mittelpunct.

Die Texte in der Laibacher Kirchenzeitung sind sowohl grammatikalisch als auch hinsichtlich der Rechtschreibung korrekt. Es kommen fast keine (Schreib)Fehler vor,

5 Alois Schlör (17. 6. 1805, Wien - 2. 11. 1852, Graz).

6 Anton Slomšek (26. 11. 1800, pri Ponikvi/zu Ponikl - 4. 9. 1862, Maribor/Marburg).

7 Jakob Maksimiljan Stepischnegg (Stepišnik) (22. 7. 1815, Celovec/Klagenfurt - 28. 6. 1899, Maribor/Marburg).

8 Peter Hitzinger (29. 6. 1812, Tržič/Neumarktl in Oberkrain - 29. 8. 1867, Postojna/Adelsberg).

9 Valentin Wiery (12. 2. 1813, Šmarno v Labotski dolini/St. Marein (im Lavanttal) - 29. 12. 1880, Celovec/Klagenfurt).

10 Leon Stanislav Vončina, (7. 4. 1826, Novi Vinodolski - 4. 11. 1874, Ljubljana/Laibach). 
das einzige, was das Lesen (neben dem komplizierten, oft philosophischen Inhalt) erschwert, ist der komplexe Satzbau mit koordinierenden oder subordinierenden Sätzen.

Aufgrund des anspruchsvolleren Inhalts und komplexeren Textaufbaus muss davon ausgegangen werden, dass das Lesepublikum der Laibacher Kirchenzeitung unter den damaligen Intellektuellen zu suchen ist. Der einfache Mensch hatte in seinem täglichen Kampf ums Überleben zum Lesen solcher Texte kein Wissen und vermutlich auch kein Interesse.

\subsection{Die Cillier Zeitung}

Der verantwortliche Redakteur der Cillier Zeitung war Vincenc Praš, Professor am hiesigen Gymnasium, der gleichzeitig auch der ständige Korrespondent von dem zuerst in Triest, später in Wien herausgegebenen Journal des oesterreichischen Lloyd war.

Im Vergleich zu der Laibacher Kirchenzeitung fällt das Gesamtbild der Cillier Zeitung unterschiedlich aus. Auf der einen Seite erschienen in der Cillier Zeitung neben Leitartikeln auch zahlreiche Nachrichten und Informationen unterschiedlicher Korrespondenten aus verschiedenen Teilen der Monarchie, die oft nur mit ihren Anfangsbuchstaben unterzeichneten. Auf der anderen Seite waren auch die Autoren der Leitartikel nicht so zahlreich. Genau genommen ging es eigentlich nur um einen ständigen Autor, Julius Galba, aus Fürstenfeld. ${ }^{11}$ Er war Student des Joaneums ${ }^{12}$ in Graz (Göth 1861: 301-303), später Secretär der Cummunität in Vordernberg (Gatti 1850: 143) und im Jahr 1848 Supplent der politischen Lehrkanzel am Theresianum ${ }^{13}$ (Galba 1848a: 189). Kurz nach der Revolution musste er das Theresianum verlassen (o. V., Wiener Zeitung, 3. 12. 1850: 3651) und wurde später Bureauchef - Stellvertreter u. Rechtsconsulent der k. k. Staats-Eisenbahn Gesellschaft (Göth 1861: 305).

Da Galba Autor der meisten Leitartikel war, bezieht sich unsere Analyse auf seine Artikel. Seiner Ausbildung ist es zu verdanken, dass seine Texte grammatisch fehlerfrei sind. Der Satzbau ist komplex, was folgender Auszug aus dem Artikel Oesterreich und seine Verfassung bestätigt (Galba 1848c: 109, vgl. Cvirn 1998: 68-69):

Österreich wird daher nur dann die Wohlfahrt seiner Völker auf die Dauer begründen, wenn es den gemeinsamen Reichstag in Wien nur über die wahrhaft gemeinsamen Angelegenheiten wie Handel, Münze, Maß und Gewicht, Heerwesen und die Vertretung nach Außen entscheiden läßt, dagegen die Gesetzgebung über jene Angelegenheiten, welche durch nationale Eigenthümlichkeiten wie die Kirchen und Schulformen, die Gerichtsverfassung, die Besteuerung, die Ablösung der Urbarialien bedingt sind, besondern Nationalparlamenten in Prag, Lemberg, Wien, Laibach, Agram und Pest einräumt.

11 Nach der Mitteilung von Prof. Dr. Norbert Allmer wurde mit seinem Vollnamen Julius Anton Galba, am 10. Juli 1821 um drei Uhr in Grazervorstadt geboren.

12 Es geht um das erste öffentliche Museum Österreichs, das im Jahr 1811 vom Erzherzog Johann von Österreich (1782-1859), dem Bruder des österreichischen Kaisers Franz I., gemeinsam mit den Ständen der Steiermark gegründet wurde.

13 Öffentliches Gymnasium der Stiftung Theresianische Akademie in Wien. 
$\mathrm{Da}$ in Galbas Leitartikeln grammatische Fehler fast nie vorkommen, betrachten wir den nächsten Textauszug aus demselben Leitartikel aus der Perspektive der Rechtschreibung, die im 19. Jahrhundert doch anders aussah (Galba 1848c: 110):

\begin{abstract}
Würde Österreich als Bundesstaat nach dem nationalen Prinzipe constituirt, so müßten Steiermark und Kärnten durchschnitten werden. Der nördlich von der Drau gelegene Theil Steiermarks und Kärntens müßte sich mit Ober und Niederösterreich und dem größeren Theile Tirols, der südlich von der Drau gelegene Theil Steiermarks und Kärtners dagegen müßte sich mit Krain und Küstenlande vereinigen, weil der Draufluß die durchschnittliche Gränze der deutschen und slovenischen Bevölkerung bildet.
\end{abstract}

Die für das 19. Jahrhundert übliche Fraktur und das Schriftzeichen „,““ werden an dieser Stelle nur erwähnt, die Ausdrücke Prinzipe und Theil werden jedoch näher betrachtet. Bei Prinzipe geht es um das Flexionsallomorph (-e) im Dativ (kurz Dativ-e), was in der damaligen Zeit bei Maskulina und Neutra der starken Kasusflexion die obligatorische Endung war. Im heutigen Deutsch wird es nur noch fakultativ bzw. selten verwendet. Im mündlichen Sprachgebrauch ist die Dativendung im Zuge des umfassenden Prozesses der e-Apokope obsolet geworden. Sie verschwindet bis zum 16. Jahrhundert im Oberdeutschen beinahe vollständig, größtenteils im Westmitteldeutschen und teilweise im Ostmitteldeutschen. Zwar nimmt seit dem sechzehnten Jahrhundert der Gebrauch des Dativ-e ausgehend vom Ostmitteldeutschen wieder zu, bleibt jedoch in weiten Teilen fakultativ. Im Schulunterricht wurde dieses Dativ-e bis vor wenigen Generationen allerdings noch streng verlangt, sodass es in der Schriftsprache bis etwa 1940 noch oft benutzt wurde (Solms/Wegera 2000: 1543). Bei Theil, das heute als Teil geschrieben wird, geht es um die alte Schreibform des Wortes, denn noch die vierte Auflage des Duden aus dem Jahr 1895 schrieb vor, dass einige Wörter wie tun oder Tür mit th geschrieben werden müssen. Teil durfte zwar als Theil oder Teil geschrieben werden, jedoch wurde in allen analysierten Zeitungen Theil geschrieben. Der Grund dafür liegt in der Tatsache, dass es im 19. Jahrhundert noch keine für den gesamten deutschen Sprachraum verbindliche Rechtschreibung gab. Bestrebungen hinsichtlich einer einheitlichen Orthographie gab es viel später. So fand z. B. die Erste staatliche Konferenz „zur Herstellung größerer Einigkeit in der Rechtschreibung“ in Berlin erst im Jahr 1874 Jahre statt. ${ }^{14}$

Im Hinblick auf den Wortschatz wurden bei Galba einige Merkmale gefunden, die unsere Aufmerksamkeit erregten. So sehen wir beispielsweise, dass sein Schreiben manchmal durch das österreichische Milieu geprägt wurde wie beim Ausdruck Naderern (,Spitzel`) (Galba 1848d: 106).

Beim Ausdruck Fassionen (,Steuererklärung') (Galba 1848g: 117), dessen Herkunft lateinisch-mittellateinisch ist, ist einerseits der Einfluss seiner Ausbildung bzw. seines Wissens spürbar. Andererseits handelt es sich dabei wahrscheinlich um einen

14 https://www.duden.de/ueber_duden/geschichte-der-rechtschreibung [08.02.2019]. 
für diese Zeit neuen, moderneren Ausdruck, für den es im Deutschen noch keinen relevanten Ausdruck gab bzw. es wurde lieber ein üblicheres Wort dafür benutzt. Es handelte sich nämlich um einen oft vorkommenden Begriff der Gesetzgebung des 19. Jahrhunderts. ${ }^{15}$

In dem Titel des Artikels Die strafbare Ehrenverletzung (Galba $1848 \mathrm{ab}$ ) kann man das Wort Ehrenverletzung nicht (mehr) im modernen Duden-Wörterbuch finden. Es ist interessant, dass es auch nicht in den alten Wörterbüchern von Lexer und Adelung zu finden ist, dagegen aber noch im Wörterbuch von Jacob und Wilhelm Grimm (1862: 66) und in den alten deutsch-slowenischen Wörterbüchern von Wolf (1860: 379) und Janežič (1889: 155).

Schließlich sollte noch eine Seltenheit in Galbas Artikeln erwähnt werden, der man weder in der Laibacher Kirchenzeitung noch im Sloveniens Blatt begegnet. Es geht um die Form der im September 1848 erschienenen Leitartikel Das alte und das neue Sistem (Galba 1848h) und Die Bierkneipe (Galba 1848ef). Galba greift hier zum Dialog bzw. Trialog. Gewöhnlich wurden mithilfe dieser Formen Probleme oder Erkenntnisse erörtert und zwar im Sinne der klassischen Dialektik, das heißt These und Antithese. Diese Formen benutzte Janez Bleiweis ${ }^{16}$ (Gjurin 1983). Galba versuchte in den erwähnten Artikeln den Lesern die neuen Erscheinungen und Errungenschaften in der Politik und Gesellschaft näher zu erläutern.

\subsection{Das Sloveniens Blatt}

Ausnahmsweise sollten zunächst die Verhältnisse, unter denen diese Zeitung erschien, beschrieben werden. Es scheint nämlich, dass die Publikation durch ihr Milieu stärker als die anderen analysierten Zeitungen geprägt wurde. Die Verhältnisse, unter denen das Sloveniens Blatt herausgegeben wurde, unterschieden sich deutlich von denen in Ljubljana (Laibach) oder Celje (Cilli). Die Stadt Novo mesto (Neustadtl) war viel kleiner, mit 3.000 Einwohnern, vorwiegend Händlern und Handwerkern, die sich fast alle auch mit der Landwirtschaft befassten (vgl. Jakopec 1990). In der Stadt lebten 60 Beamte, die ausschließlich Deutsch sprachen, ansonsten waren die Bürger slowenischer Herkunft, die Deutsch lernen mussten. Was die Sprache angeht, waren die Verhältnisse ähnlich wie in Celje (Cilli), wo die Bürger auch deutsch sprachen. Den Unterschied merkte man aber bei ihrer nationalen Orientierung, denn in Novo mesto (Neustadtl) waren alle slowenisch orientiert, in Celje (Cilli) aber nicht (vgl. Jakopec 1994: 58).

Der verantwortliche Redakteur des Sloveniens Blattes war Franc Polak. ${ }^{17}$ Die Zeitung aus Novo mesto (Neustadtl) hatte zwar keine ständigen Kolumnisten, unter ihnen

15 Ein Beispiel dafür aus dem Literaturverzeichnis des Franziszeischen Katasters: B e 1 e h r u n g für die Herrschaften, Gutsbesitzer, Magistrate, Freysassen und alle jene, welche sonst noch zu Urbarial-Nutzungen und Jurisdictions-Gebühren berechtigt sind über die nach dem Circulare vom 1. May 1819 einzureichenden Fassionen ihre 18 Urbarial Nutzungen und JurisdictionsGebühren. (http://www.bev.gv.at 8. 2. 2019)

16 Janez Bleiweis (19. 11. 1808, Kranj/Krain - 29. 11. 1881, Ljubljana/Laibach).

17 Franc Polak (26. 3. 1815, Kranj/Krain - unbekannt). (Vgl. Jakopec 1991b.) 
finden wir bekannte Persönlichkeiten: den Kreisarzt Dr. Julius Vest, den Apotheker Franz Xaver Gadner, den Theologen Franz Xaver Richter ${ }^{18}$.

Viele Leitartikel wurden jedoch ohne Angabe des Autors veröffentlicht bzw. sie wurden nur mit Anfangsbuchstaben unterzeichnet, was die Identifizierung erschwerte. Das ist für unsere Analyse eine bedeutende Tatsache, denn es scheint, dass gerade diese Leitartikel sprachlich die meisten Fehler aufweisen.

Die Leitartikel waren in der Regel lang, oft mit mehreren Fortsetzungen, inhaltlich den beiden anderen analysierten Zeitungen ähnlich. Doch gab es auch einen deutlichen Unterschied zu den anderen beiden untersuchten Zeitungen: das Sloveniens Blatt zeigte auch Interesse an Bauern, Arbeitern und an der slowenischen Sprache.

Es scheint, dass im Vergleich zu den anderen analysierten Zeitungen das Sloveniens Blatt eine niedrige Qualität auszeichnete. Die auffallend fehlerhafte Sprache und Schreibweise im Sloveniens Blatt ist bereits im 19. Jahrhundert vermerkt worden (Apih 1888: 147).

Diese Feststellung kann unsere Analyse auch bestätigen. Obwohl es unter den Leitartikeln auch fehlerfreie Texte gab, ist anzumerken, dass diese Zeitung sprachlich auf keinem hohen Niveau war.

Aus morphologischer Sicht kam es oft zu Buchstabenauslassungen, wie die Beispiele aus den Artikeln verdeutlichen, deren Autoren unbekannt sind: uniformirten statt uniformiert (o. V. 4. 7. 1848: 4), Eintrit statt Eintritt (o. V. 19. 12. 1848: 98) oder Volkssourainät statt Volkssouveränität (o. V. 15. 8. 1848: 28). Bei dem letzten Ausdruck kann es sich vielleicht nicht nur um einen Schreibfehler handeln, sondern um ein Wort, das in der damaligen Zeit neu war und somit nicht üblich.

Bei Merz statt März (o. V. 15. 8. 1848: 28) könnte es sich auch um das Auslassen von „a“ (Maerz) handeln oder das Wort wurde phonetisch geschrieben.

Bei lies't statt liest (o. V. 24. 8. 1848: 66) geht es um einen Tippfehler. Beim Wort Eiland statt Insel (Polak 1848: 9) handelt es sich um einen Anglizismus.

Der Satzbau der Texte ist oft kompliziert und schwer verständlich, jedoch aus einem anderen Grund als bei der Laibacher Kirchenzeitung. Die Sätze im Sloveniens Blatt sind komplex und mit häufigerer Hypotaxe und korrekter Kommasetzung. Meistens tauchen jedoch Fehler bei der Wortfolge im Nebensatz auf.

Das Artikellesen im Sloveniens Blatt verlangt dem Leser größere Konzentration ab, nicht nur wegen des komplexen Satzbaues. Um etwas näher und deutlicher zu erklären, benutzten die Autoren häufig Parenthesen, was das Lesen nicht erleichtert, sondern im Gegenteil eher erschwert.

In langen Sätzen geht es oft um eine reine Aufzählung der Gedanken nach dem assoziativen Prinzip. Ein Beispiel aus dem Artikel eines unbekannten M., der über die Bauern schreibt, soll dies verdeutlichen (M. 1848: 22):

Es liegt nicht in der Absicht dieses Aufsatzes die practische Durchführung der vorgeschlagenen Maßregel zu erörtern; es möge jedoch die Bemerkung Platz

18 Franz Xaver Johann Richter (18. 8. 1783, Osoblahi/Hotzenploz - 24. 5. 1856, Wien). 
greifen, daß bei der Regulierung und Richtigstellung der Urbarialien der damit betrauten Commission ein weitgrößerer Spielraum der eigenen Thätigkeit gebothen, das Geschäft vereinfacht, den Grundsätzen des Rechtes und der Billigkeit genauere Rechnung getragen wird bei einem Verfahren, welches nicht inmitten sondern über der Partheifrage die Gefahr glücklich vermeidet, zwischen dem in der Natur der Sache gelegenen Bestreben der Gutsherren von ihren Ansprüchen so viel als möglich, und sei es das Unhaltbarste, zu hakten, und jenem der Grundholden, so wenig als möglich zuzugestehen, den eigentlichen Richtpunct aus dem Auge zu verlieren.

Es kann angenommen werden, dass die Herkunft des Autors nicht deutsch sein könnte, denn die Aussagen folgen dem assoziativen Prinzip, und der Sinn der Aussage bleibt undeutlich. Und hier kommen wir zu der erwähnten Tatsache zurück, dass das (slowenische) Milieu die Zeitung inhaltlich und auch sprachlich stark beeinflusste.

In der Zeitung geht es zudem auch noch um technische Fehler wie z. B. in der letzten Nummer, in der angegeben wurde, dass die nächste Nummer wegen den Weihnachtsferien erst am Mittwoch, den 29. herausgegeben wird. Der 29. Dezember fiel jedoch auf einen Freitag und nicht auf einen Mittwoch (Granda 1994: 232-233).

Will man die Gründe für den spezifischen Gebrauch der (deutschen) Sprache im Sloveniens Blatt zusammenfassen, so müssen mehrere Faktoren berücksichtigt werden. Einerseits liegen die Ursachen für das niedrigere Sprachniveau wahrscheinlich in der Tatsache begründet, dass die unbekannten Autoren der Leitartikel keine hochgebildeten Intellektuellen waren. Es könnte sein, dass sie ihre Ideen einfach direkt aus dem Slowenischen ins Deutsche übersetzten, ohne dabei an die Regeln der deutschen Grammatik gedacht zu haben. Diese Vermutung liegt nahe, weil es sich bei den Leitartikeln bekannter Autoren in der Regel um fehlerfreie Texte handelt.

Andererseits aber sollte auch das Milieu berücksichtigt werden, das die Sprache der Zeitung prägte, da im Unterschied zu den anderen analysierten Zeitungen die Stadt und ihre Umgebung überwiegend slowenisch orientiert waren.

\section{SCHLUSS}

Die Aufhebung der Zensur ermöglicht eine Blütezeit des Zeitungswesens. Überall in der Monarchie erschienen neue Zeitungen, so auch in den Ländern mit slowenischer Bevölkerung. Hier war die deutsche Sprache den gebildeten Bürgern vertrauter und geläufiger als Slowenisch, das damals keinen angemessenen Wortschatz für Diskussionen über die neuen politischen Themen aufwies. Die deutsche Sprache der analysierten Zeitungen ist also kein Zeichen der Abwendung vom Slowenentum, sondern sie ermöglichte es, das damals moderne Gedankengut auszudrücken. Einige Zeitungen, und das gilt besonders für Sloveniens Blatt, sind trotz der deutschen Sprache völlig vom Geist des Slowenentums geprägt. Einige Sätze weisen auf die slowenische Denkweise hin, was das Verstehen erschwert. Die anderen analysierten Zeitungen sind morphologisch und syntaktisch korrekter und anspruchsvoller. Der Grund dafür liegt bestimmt auch in der Tatsache, dass die Autoren der Leitartikel der Laibacher Kirchenzeitung und der Cillier Zeitung vor allem in Wien 
ausgebildete Persönlichkeiten waren. Es ist bekannt, dass die Ausbildung (und somit das Lesen) die Schreibkompetenz verbessert und den eigenen Schreibstil beeinflusst.

Auch das Lesepublikum der untersuchten Zeitungen war verschieden. Die Laibacher Kirchenzeitung wurde dank ihrer Orientierung und des komplexen Inhaltes eher von einem engeren Publikum (Kirche) rezipiert, die anderen zwei jedoch von einem breiteren Kreis der (gebildeten) Bürger.

\section{Primärliteratur}

Cillier Zeitung (1848). Nr. 21, 22, 24, 35, 39, 40, 42, 45.

GALBA, Julius (1848a) „Die strafbare Ehrenverletzung.“ Cillier Zeitung, 6. 10. 1848, $42,189$.

GALBA, Julius (1848b) „Die strafbare Ehrenverletzung.“ Cillier Zeitung, 17. 10. 1848, 45, 201-202.

GALBA, Julius (1848c) „Österreich und seine Verfassung.“ Cillier Zeitung, 28. 7. $1848,22,109-110$.

GALBA, Julius (1848d) „Metternich und das alte Sistem.“ Cillier Zeitung, 25. 7. 1848, $21,106$.

GALBA, Julius (1848e) „Die Bierkneipe.“ Cillier Zeitung, 26. 9. 1848, 39, 177-178.

GALBA, Julius (1848f) „Die Bierkneipe.“ Cillier Zeitung, 29. 9. 1848, 40, 181-182.

GALBA, Julius (1848g) „Die Einkommenssteuer.“ Cillier Zeitung, 4. 8. 1848, 24, 117.

GALBA, Julius (1848h) „Das alte und das neue Sistem.“ Cillier Zeitung, 12. 9. 1848, 35, 161-162.

HITZINGER, Peter (1848) „Einige Worte über die künftige Stellung der Kirche.“ Laibacher Kirchenzeitung, 3. 8. 1848, 5, 33.

Laibacher Kirchenzeitung (1848). 1, 2, 21.

Laibacher Zeitung (1848). Nr. 5.

M. (1848) „Die Urbarialfrage.“ Sloveniens Blatt, 8. 8. 1848, 6, 22.

o. V. (1848) „Die Nationalgarde in Neustadt1.“ Sloveniens Blatt, 4. 7. 1848, 1, 4.

o. V. (1848) Sloveniens Blatt, 19. 12. 1848, 25, 98.

o. V. (1848) „Wien gegenüber dem Provinzialismus.“ Sloveniens Blatt, 15. 8. 1848, 7, $27-28$.

o. V. (1848) „Wie stehen die Partheien in Oesterreich?“ Sloveniens Blatt, 24. 8. 1848, $17,66$.

o. V. (1850) Wiener Zeitung, 3. 12. 1850, 3651.

POGAZHAR, Janez (1848b) „Was jetzt Noth thut.“ Laibacher Kirchenzeitung, 13. 7. $1848,2,10$.

POGAZHAR, Janez (1848a) „Was jetzt Noth thut?“ Laibacher Kirchenzeitung, 6. 6. $1848,1,1$.

POLAK, Franc (1848) „Die Revolution im Jahre 1848،“ Sloveniens Blatt, 18. 7. 1848, 3, 9. Sloveniens Blatt (1848). Nr. 1, 7, 17, 25.

STEPISCHNEGG, Jakob Maksimiljan (1848) „Gedanken über Proletariat, Presse und

Kirche." Laibacher Kirchenzeitung, 23. 11. 1848, 21, 161-163.

Wiener Zeitung (1848). Wien: van Ghelen'sche Erben (Nr. 288). 


\section{Sekundärliteratur}

AMBROŽIČ, Matjaž (2003) Ljubljanski knezoškof dr. Janez Zlatoust Pogačar. Ljubljana: Acta Ecclesiastica Sloveniae.

APIH, Joseph (1888) Slovenci in leto 1848. Ljubljana: Matica slovenska.

CANKAR, Izidor/Franc Ksaver LUKMAN/Franc KIDRIČ/Alfonz GSPAN/Fran PETRÈ/Jože MUNDA (Hrsg.) (1925-1991) Slovenski biografski leksikon. Ljubljana: Zadružna gospodarska banka.

CVIRN, Janez (1998) „Celje in leto 1848.“ In: M. Počivavšek et al. (Hrsg.), Iz zgodovine Celja 2, 59-72.

GATTI, Ferdinand (1850) Die Ereignisse des Jahres 1848 in der Steiermark. Graz: In Commission bei J. Kienreich.

GJURIN, Velemir (1983) „Bleiweisov poljudnostrokovni jezik.” In: J. Batis/P. Vodopivec (Hrsg.), Zbornik za zgodovino naravoslovja in tehnike 7: Bleiweisov zbornik. Ljubljana: Slovenska matica, 241-259.

GÖTH Georg (1861) Das Joanneum in Gratz, geschichtlich dargestellt zur Erinnerung an seine Gründung vor 50 Jahren. Gratz: Leykam.

GRANDA, Stane (1994) „Sloveniens Blatt - časopis iz leta 1848.” Zgodovinski časopis 48/2, 231-252.

GRANDA, Stane (1999) Prva odločitev Slovencev za Slovenijo: Dokumenti z uvodno študijo in osnovnimi pojasnili. Ljubljana: Nova revija.

GRIMM, Jakob/Wilhelm, GRIMM (1862) Deutsches Wörterbuch: Dritter Band. Leipzig: Verlag von Hirzel.

JAKOPEC, Miloš (1990) „Časnikarstvo v Novem mestu.“ Rast 1/2, 110-119.

JAKOPEC, Miloš (1994) Časnikarstvo na Dolenjskem 1848-1941. Novo mesto: Tiskarna Novo mesto/Dolenjska založba: Dolenjski list.

JANEŽIČ, Anton (1889) Deutsch-slowenisches Wörterbuch. Klagenfurt: St. Hermagoras-Bruderschaft.

MILADINOVIĆ ZALAZNIK, Mira/Stane GRANDA (Hrsg.) (2009) Anton Aleksander grof Auersperg - Anastazij Grün: razprave. Ljubljana: Nova revija.

PETERNEL, Marija Mojca, (2006) Cillier Wochenblatt (Zeitung): Celjski nemški časopis iz leta 1848. Celje: Zgodovinsko društvo.

PETERNEL, Marija Mojca (2015) „Die Laibacher Kirchenzeitung: Pressefreiheit und Kirche im Revolutionsjahr 1848.“ Blätter für Heimatkunde 89/1-2, 25-41.

POCK, Friedrich (1925) „Von der deutschen Presse in Untersteier.“ In: F. Hausmann (Hrsg.), Südsteiermark. Ein Gedenkbuch. Graz: Verlag Ulr Mosers Buchhandlung, 182-189.

SOLMS, Hans-Joachim/Klaus-Peter WEGERA (2000) „Morphologie des Frühneuhochdeutschen." In: W. Besch/A. Betten/O. Reichmann/S. Sonderegger (Hrsg.), Sprachgeschichte: Ein Handbuch zur Geschichte der deutschen Sprache und ihrer Erforschung. 2., vollständig neu bearbeitete und erweiterte Auflage. Berlin/New York: Walter de Gruyter, 1542-1553.

WOLF, Anton Alois (1860) Deutsch-slowenisches Wörterbuch. Laibach: Joseph Blaznik. 
WURZBACH, Constantin von (1856/1891) Biographisches Lexikon des Kaiserthums Oesterreich, enthaltend die Lebensskizzen der denkwürdigen Personen, welche 1750 bis 1850 im Kaiserstaate und seinen Kronländern gelebt haben. Wien: L. C. Zamarski/Kaiserlich-königlicher Hof- und Staatsdruckerei.

ZENKER, Ernst Victor (1893) Geschichte der Wiener Journalistik: ein Beitrag zur deutschen Culturgeschichte. I-II. Wien/Leipzig: W. Braumüller.

\section{Internetquellen}

Mittelhochdeutsches Handwörterbuch von Matthias Lexer. http://woerterbuchnetz.de/ cgi-bin/WBNetz/wbgui_py?sigle=Lexer [12.12.2018].

Adelung - Grammatisch-kritisches Wörterbuch der Hochdeutschen Mundart. https:// lexika.digitale-sammlungen.de/adelung/suche/ [10.01.2019].

Duden Wörterbuch. https://www.duden.de [13.01.2019].

Deutsches Wörterbuch von Jacob Grimm und Wilhelm Grimm: http://woerterbuchnetz. de/cgi-bin/WBNetz/wbgui_py?sigle=DWB [22. 1. 2019].

\section{Zusammenfassung \\ DEUTSCH IN DER PRESSE UM 1848 IN DEN VON SLOWENEN BESIEDELTEN GEBIETEN: EINE EXEMPLARISCHE ANALYSE AM BEISPIEL VON LAIBACHER KIRCHENZEITUNG, CILLIER ZEITUNG UND SLOVENIENS BLATT}

Im Beitrag wurde versucht, die durch die Revolution 1848/49 initiierten deutschen Zeitungen (das heißt neue Zeitungen) im Raum mit slowenischer Bevölkerung aus sprachlicher Perspektive näher zu erläutern und zu vergleichen. Da das Jahr 1848/49 durch die Revolution geprägt wurde, und die Zeitungen Spiegel ihrer Zeit sind, trugen diese einen ausgeprägt politischen Charakter. In diesem Zusammenhang wollten wir recherchieren, in welchem Ausmaß die Sprache der neuen Zeitungen dadurch geprägt worden sind.

Es wurde versucht, vor allem auf der morphologischen und syntaktischen Ebene Merkmale bzw. eventuelle Unterschiede im Sprachgebrauch festzustellen und nach deren Ursache zu fragen. Es wurde festgestellt, dass die analysierten Zeitungen sprachliche Unterschiede aufweisen. Die Gründe dafür fanden wir auf der einen Seite bei den Autoren der Leitartikel, und zwar besonders bei ihrer Ausbildung, und auf der anderen Seite beim Lesepublikum bzw. im Milieu, in dem die Zeitungen herausgegeben wurden. Kein Wunder also, das Sloveniens Blatt im Vergleich zu der Cillier Zeitung wie auch Laibacher Kirchenzeitung trotz der deutschen Sprache völlig vom Geist des Slowenentums geprägt war. Die Recherche bewies auch die am Anfang gestellte Hypothese, dass die Zeitungssprache durch das Milieu, in dem man sie herausgab, beeinflusst wurde.

Schlüsselwörter: deutsche Zeitungen, Revolution 1848/49, deutsche Sprache, Geschichte 


\section{Abstract \\ GERMAN IN THE PRESS AROUND 1848 IN THE AREAS POPULATED BY \\ SLOVENES: AN EXEMPLARY ANALYSIS OF LAIBACHER KIRCHENZEITUNG, CILLIER ZEITUNG AND SLOVENIENS BLATT}

The article tries to explain and compare the German newspapers which started to be published in the revolutionary year 1848/49 in areas where a Slovenian-speaking population was present. Since the year 1848/49 was marked by revolution and the newspapers are mirrors of their time, it is not surprising that they had a pronounced political character. In this context we wanted to explore the extent to which the language of the new newspapers has been shaped by the external circumstances.

We sought to identify the characteristics of and possible differences in language use, particularly on the morphological and syntactic levels, and to investigate the possible reasons behind it. Our analysis has shown that the newspapers do present linguistic differences, which may be related to different educational backgrounds of the authors as well as to the specific readerships and the social milieus in which the newspapers were published. It is not surprsing then that Sloveniens Blatt in comparison with Cillier Zeitung and Laibacher Kirchenzeitung was influenced by a Slovenian nationaistl spirit despite being a German language publication.

Keywords: German newspapers, Revolution 1848/49, German language, history

Povzetek

NEMŠČINA V ČASOPISIH OKOLI LETA 1848 NA OBMOČJIH

S SLOVENSKIM PREBIVALSTVOM: PRIMERJALNA

ANALIZA ČASOPISOV LAIBACHER KIRCHENZEITUNG, CILLIER ZEITUNG AND SLOVENIENS BLATT

Prispevek predstavlja analizo nemških časopisov, ki so se pojavili z revolucijo leta 1848/49, z jezikovnega vidika. Ker je revolucionarno leto 1848/49 politično leto in ker so časopisi ogledalo časa, $v$ katerem izhajajo, imajo vsi analizirani časopisi izrazito politični karakter. Pri analizi smo poskušali ugotoviti, v kakšni meri je to dejstvo vplivalo na jezikovno podobo časopisa.

Poskušali smo ugotoviti, ali obstajajo razlike med posameznimi časopisi ter kakšni so razlogi za njihov nastanek. Jezikovna analiza nemških časopisov se je dotaknila predvsem morfološke in sintaktične ravnine jezika. Ugotovili smo, da se časopisi med seboj jezikovno razlikujejo in da so razlike nastale predvsem iz dveh različnih razlogov. Na eni strani so k temu pripomogli avtorji člankov, zlasti njihova izobrazba, na drugi strani pa okolje, v katerem je posamezen časopis izhajal, oziroma njegova ciljna publika. Tako je bil časopis Sloveniens Blatt v primerjavi s Cillier Zeitung in Laibacher Kirchenzeitung po pričakovanju jezikovno šibkejši, ne le zaradi vsebine člankov, temveč predvsem zaradi ciljne publike oziroma miljeja, v katerem je izhajal. S tem je 
je bila potrjena hipoteza, da se je tudi okolje, v katerem so časopisi izhajali, zrcalilo v jezikovnih značilnostih posameznega časopisa.

Ključne besede: nemški časopisi, revolucija leta 1848/49, nemški jezik, zgodovina 